\title{
Subject-specific body segment parameter estimation using 3D photogrammetry with multiple cameras
}

Kathrin E Peyer, Mark Morris, William I Sellers

Inertial properties of body segments, such as mass, centre of mass or moments of inertia, are important parameters when studying movements of the human body. These quantities are, however, not directly measurable. Current approaches include using regression models which have limited accuracy; geometric models with lengthy measuring procedures; or acquiring and post-processing MRI scans of participants. We propose a geometric methodology based on 3D photogrammetry using multiple cameras to provide subject-specific body segment parameters while minimizing the interaction time with the participants. A low-cost body scanner was built using multiple cameras and 3D point cloud data generated using structure from motion photogrammetric reconstruction algorithms. The point cloud was manually separated into body segments and convex hulling applied to each segment to produce the required geometric outlines. The accuracy of the method can be adjusted by choosing the number of subdivisions of the body segments. The body segment parameters of six participants (four male and two female) are presented using the proposed method. The multi-camera photogrammetric approach is expected to be particularly suited for studies including populations for which regression models are not available in literature and where other geometric techniques or MRI scanning are not applicable due to time or ethical constraints. 


\section{Authors}

2

3 Kathrin Eva Peyer ${ }^{1}$, Mark Morris ${ }^{1}$, William Irvin Sellers ${ }^{1}$

4

$5 \quad{ }^{1}$ Faculty of Life Sciences, University of Manchester, Manchester, United Kingdom 


\section{1. Introduction}

11 Inertial body segment parameters (BSP), such as mass, centre of mass (CoM) or moment of inertia, are used in motion analysis in research as well as in clinical settings. Accurate values are essential for techniques such as inverse dynamic analysis to allow the calculation of joint torques based on measured segmental accelerations (Winter, 1979). It is, however, not straightforward to measure these quantities from subjects directly. One approach is to use mathematical models of the body segments and rely on anthropometric measurements to determine the dimensions of the modelled segments. This type of methods requires a multitude of anthropometric measurements of the participants and is limited by the accuracy of the mathematical model of the body segments. The first mathematical model suggested by Hanavan in 1964 represented 15 body segments as cylinders and spheres and required 25 anthropometric measurements (Hanavan, 1964). More detailed models presented by Hatze or Yeadon required a total of 95 or 242 measurements respectively rendering these methods inefficient for studies with a large number of participants because of the time and discomfort experienced by the participant to acquire all the measurements needed (Hatze, 1980; Yeadon, 1990). Other types of approaches rely on X-ray or MRI based tomography to extract subject-specific BSP from participants. Unlike other methods, CT or MRI scans provide information about internal structures such as tissue composition which should improve the reconstruction accuracy (Martin et al., 1989; Mungiole \& Martin, 1990; Pearsall, Reid \& Livingston, 1996; Bauer et al., 2007). These approaches are, however, also difficult to implement in large-scale studies due to cost and ethical constraints. Alternatively, it is possible to approximate inertial BSP by adjusting previously reported average values or using regression models that require only a very few subject-specific measurements (commonly subject height and weight). Such average values and regression models were derived from cadavers or participants in a number of famous studies, such as the ones by Clauser, Dempster or Zatsiorsky (via de Leva) (Dempster, 1955; Clauser, McConville \& Young, 1969; McConville, Clauser \& Churchill, 1980; Leva, 1996). The reliability of such regression models is, however, rather low and the models are only applicable to a population similar to the one used to derive the regression equations.

Recently, other methods have been explored to obtain volumetric data of body segments that, in combination with body density assumptions, can provide subject-specific inertial BSP. Sheets et 
42 contained joint location information, to the scanned surface (Sheets, Corazza \& Andriacchi,

43 2010). Bonnechere et al used a Kinect sensor to estimate body segment lengths but not the

44 volumetric data required to estimate inertial properties (Bonnechère et al., 2014). Clarkson

45 evaluated the Kinect sensor as a surface scanner using a mannequin, but found the scanning

46 resolution to be quite low (Clarkson et al., 2012). Another approach to gain surface data is to use

47 photogrammetry. In 1978, Jensen proposed the use of stereophotogrammetry to estimate BSP

48 parameters (Jensen, 1978). In his model, the human body was divided into elliptical disks with a

49 thickness of $20 \mathrm{~mm}$ and the radii of the elliptical disks were estimated using images from the

50 front and side. The drawback of this approach lies in the simplifying assumptions of representing

51 body segments as the elliptical disks. It is, however, possible to reconstruct the surface of a 3D

52 object from multiple uncalibrated 2D images taken from different positions without requiring

53 any assumptions to the geometry of the body. This principle is referred to as "structure from

54 motion" and was initially used for producing 3D models of static objects and landscapes.

55 Perhaps the most striking example to date is the "Building Rome in a Day" project which used

56 images from the Flikr web site (http://www.flickr.com) to generate a 3D model of the whole city

57 (Agarwal et al., 2009). The reconstruction of a 3D surface from multiple cameras is two-stage

58 process. In stage one, the position, orientation and the parameters of the camera optics are

59 estimated. This is achieved by the bundle adjustment algorithm (Triggs et al., 2000) that

60 minimizes the error between the re-projected feature points using estimated camera pose and

61 parameters with the actual feature points in the images. In theory, feature points could be chosen

62 manually but this would be cumbersome and potentially not very accurate. Instead, Scale

63 Invariant Feature Transform (SIFT) algorithms are employed which automate this process by

64 identifying possible common points between multiple images (Lowe, 1999). Stage two uses the

65 calibrated views to produce a dense point cloud model of the 3D object. There are a number of

66 possible approaches to achieve this (for review see (Seitz et al., 2006)) but probably the most

67 widespread current approach is patch-based multi-view stereo reconstruction (Furukawa \&

68 Ponce, 2010). This photogrammetric approach has gained wide acceptance for producing 3D

69 models in areas such as archaeology (McCarthy, 2014) and palaeontology (Falkingham, 2012),

70 and is even used for markerless motion capture (Sellers \& Hirasaki, 2014).

71 The aim of this paper is to investigate whether an approach based on structure form motion

72 photogrammetric reconstruction can provide person-specific body segment parameters and to

73 identify the strength and weaknesses of such an approach with regards to ease of

74 implementation, cost-effectiveness, subject comfort and processing time. A low-cost body 


\section{PeerJ Reviewing Manuscript}

75

76

77

78

79

80

81

82

scanner was built using multiple cameras and the body segment parameters of six participants (four male and two female) are presented using the proposed method.

\section{Methods}

Photogrammetry relies on obtaining multiple photographs taken from different locations. These photographs can be taken with any suitable device and for objects that do not move, the most cost effective option is to take $50+$ photographs with a single camera that is moved around the object. This has the additional advantage that a single intrinsic calibration can be used since the camera optics can be considered identical for multiple images. However for subjects that can move, all the photographs must be taken simultaneously so that the subject is in exactly the same position for all the images. Simultaneous photographs can be achieved in several different ways including multiple still cameras with synchronised remote controls, multiple USB web cameras, or multiple networked cameras. There is probably little to choose between these methods but initial experimentation found that network/IP cameras provided a cost effective solution that scaled well. The camera resolution should be as high as reasonably possible since higher resolution images provide more information for the feature extraction algorithms and higher point density in the eventual reconstruction. This means that low resolution cameras such as low cost web cameras and standard resolution video cameras may not be suitable.

Most applications that employ photogrammetry aim to capture surface data in great detail with the emphasis on creating almost true-to-live 3D models and thus maximizing the point cloud density. Some applications require only the information available from the point cloud directly (such as feature point locations) and do not require a surface mesh. In fact, meshing algorithms tend to decrease the accuracy of the model (Falkingham, 2012). In applications where the reconstructed object is to be 3D-printed (Garsthagen; Hobson; Straub \& Kerlin, 2014) or where volumetric data is required (such as for body segment estimations presented in this paper), a (closed) surface mesh needs to be created from the point cloud. A high-resolution mesh is commonly desired in 3D printing (e.g. for aesthetic or functional reasons), which requires a large number of photographs and sophisticated algorithms to convert the point cloud to a mesh. In this paper, we propose the use of convex hulling to generate simplified geometric outlines of the body segments. Convex hulling is robust to low-density surface point clouds (and even potential gaps in the point cloud) and can thus be implemented with ease and run automatically without requiring user input. Furthermore, being able to generate surface meshes from a low-density 
108 point cloud lowers the number of cameras required to build the 3D scanner (as opposed to

109 needing a large number of cameras to achieve densely packed point clouds).

111 2.1. 3D body scanner design

112 Photogrammetric reconstruction can work well with as few as 4 cameras (Sellers \& Hirasaki,

113 2014)but more cameras are necessary to provide a relatively gap free reconstruction. To estimate

114 the minimal number of cameras necessary to achieve a $360^{\circ}$ reconstruction, we positioned a

115 single camera on a circle of radius $1.6 \mathrm{~m}$ and placed a stationary skeletal dummy as a test

116 subject in the centre.. Images were taken every $5^{\circ}$ and the point cloud reconstructions using 72 ,

$11736,24,18,12$ and 9 images, corresponding to angular resolutions ranging from $5^{\circ}$ to $40^{\circ}$, were

118 compared (see Fig. 1A). Acceptable reconstructions for the purpose of this paper, i.e. no loss of

119 body segment features, were found with 18 or more cameras although using larger numbers of

120 cameras certainly improved the point cloud density. After initial testing, the setup design was

121 adjusted by increasing the radius of the camera placements (to increase the field of view to

122 accommodate outstretched arms), placing the cameras above head-hight and angling the camera

123 views downwards (as opposed to placing the cameras at the bottom or at hip-height) and using

124 asymmetric patterns on the floor in the shared field of view of all cameras. The latter greatly

125 aided the reconstruction reliability as the camera calibration algorithm relies on shared features. ${ }^{1}$

126 The network camera was implemented using Raspberry Pi (RPi) modules, type A, each equipped

127 with an 8GB SD card and a Pi camera (http://www.raspberrypi.org). These modules run the

128 Linux operating system (Raspbian) and provide a flexible and cost-effective 5 megapixel

129 network camera platform. The $18 \mathrm{RPi}$ modules (each with a camera) were attached to a $4.8 \mathrm{~m}$

130 diameter hexagonal frame elevated to height of $2.3 \mathrm{~m}$ by six support poles (see Fig. 1B). Each

131 RPi module was provided with a USB WiFi receiver (Dynamode WL-700-RX) and power was

132 provided using the standard RPi power adapter plugged into a multi-socket attached to each

133 support pole. Four $500 \mathrm{~W}$ Halogen floodlights were mounted to provide additional lighting to

134 increase the image quality.

135

136 RPi cameras can record either still images or movie files. For this application we needed to

137 trigger all the cameras to record a single image at the same instant. This was achieved using the

138 open source "Compound Pi" application (http://compoundpi.readthedocs.org), which uses the

1 Without the patterns on the floor, the camera calibration relied on shared features found on the subject, whereas the patterned floor provided a large (or even completely sufficient) number of features to run the camera calibration algorithm. 
139 UDP broadcast protocol to control multiple cameras synchronously from a single server. Once

140 the individual images have been recorded, the application provides an interface to download all

141 the images obtained to the server in a straightforward manner. Since UDP broadcast is a one-to-

142 many protocol, all the clients will receive the same network packet at the same time and the

143 timing consistency for the images will be of the order of milliseconds which is adequate for a

144 human subject who is trying to stand still. Higher precision synchronisation can be achieved

145 using a separate synchronisation trigger but this was unnecessary in this application.

\subsection{Data acquisition}

148 Full body scans using the RPi setup were obtained from six voluntary participants. Additionally, 149 their body weight and height was measured (Table 1). The male visible human was used as an 150 additional data set for validation (National Library of Medicine's Visual Human Project (Spitzer

151 et al., 1996)). The experimental protocol (reference number 13310) was approved by the

152 University of Manchester ethics panel. In accordance with the experimental protocol, written

153 consent was obtained from all participants.

155 The reconstruction algorithms rely on finding matching points across multiple images so they do

156 not work well on images that contain no textural variation. We therefore experimented with 157 using different types of clothing in the scanner, such as sports clothing, leisure clothing, and a

158 black motion capture suit equipped with Velcro strips to aid feature detection. Clothing was

159 either body-tight or tightened using Velcro strips if they were loose since loose clothing would

160 lead to an overestimation of the body volume. The participants stood in the centre of the RPi

161 setup with their hands lifted above their head (see Fig. 2) and the 18 images were then acquired.

\subsection{Data processing}

166 The 3D point cloud reconstruction was initially done using open source application VisualSFM

167 (http://ccwu.me/vsfm/) which performed adequately, but we then switched to using Agisoft

168 PhotoScan Standard Edition v1.0.4 (http://www.agisoft.com) which proved to be much easier to 169 install and use. Agisoft PhotoScan also achieved a better reconstruction quality with fewer holes 
170 in the point cloud and smoother surfaces. ${ }^{2}$ The parameters used in the reconstructions are

171 reported in the supporting information. Agisoft PhotoScan runs identically on Windows, Mac or

172 Linux. The full 3D reconstruction with 18 images took an average of 30 minutes using an 8 core

$1733 \mathrm{GHz}$ Xeon MacPro with 12GB RAM. The actual time taken was variable depending on the

174 image file size and the reconstruction parameters. The output of the Agisoft PhotoScan is an

175 unscaled 3D point cloud of the participants and surrounding scenery (see Fig. 2), which requires

176 further post-processing to calculate BSP values. First, the point cloud was scaled and oriented

177 using CloudDigitizer (Sellers \& Hirasaki, 2014), the oriented point clouds were then divided into

178 anatomical segments using Geomagic (http://geomagic.com), and the convex hulls computed in

179 Matlab ${ }^{\circledR}$ (http://www.mathworks.com, see supporting information). The reference points for the

180 body segmentation are listed in the supporting information Table S1. The body segments were all

181 oriented into the standard anatomical pose before the volume, centre of mass and inertial tensor

182 were calculated based on the hull shape and segment density using a custom function

183 implemented in Matlab ${ }^{\circledR}$ (see supporting information). The choice of body density is an

184 interesting issue. Different tissues within segments have different densities and tissue

185 composition is moderately variable between individuals. Indeed variations in density are

186 commonly used to estimate body fat percentage (Siri, 1961; Brožek et al., 1963). MRI and CT

187 based techniques can allow individual tissue identification and can compensate for this but

188 surface volumetric techniques need to use an appropriate mean value. Segment specific densities

189 are available (e.g. (Winter, 1979)) but the quoted trunk density is after subtraction of the lung

190 volume. For a surface scan model, we need to use a lower value trunk density that incorporates

191 the volume taken up by the air within the lungs. Therefore for the purpose of this paper a trunk

192 density value of $940 \mathrm{~kg} / \mathrm{m}^{3}$ was chosen, while a uniform density of $1000 \mathrm{~kg} / \mathrm{m}^{3}$ was assumed for

193 all other body segments (Weinbach, 1938; Pearsall, Reid \& Ross, 1994). The body mass

194 calculated from the volume was never exactly the same as the recorded body mass so the density

195 values were adjusted pro-rata to produce a consistent value for total mass.

$196 \mathrm{~s}=\frac{m_{\text {Participant }}}{\sum m_{\text {SegmHull,i }}}$

197 The factor $s$ effectively scales the body densities and is thus also applied the moments and

198 products of inertia obtained from the convex hull segments (see supporting information). 199

2 This is based on the comparison of the best reconstruction result achieved with each software after testing an extensive, but not complete, combination of reconstruction parameters. 
202 Six participants were scanned using the RPi photogrammetry setup and their point cloud 203 segmented. In order to be able to calculate the inertial properties, the point cloud needs to be 204 converted into a closed surface mesh. To calculate the volume of an arbitrary shape defined by a 205 surface mesh, the mesh needs to be well defined, i.e., it should be two-manifold, contain no holes 206 in the mesh, and have coherent face orientations. The processing of converting a point cloud to a 207 well defined mesh is known as hulling and there are several possible methods available. The 208 simplest is the minimum convex hull where the minimum volume convex shape is derived mathematically from the point cloud (www.qhull.org). This approach has the advantage of being extremely quick and easy to perform and it is very tolerant of point clouds that may contain holes where the reconstruction algorithm has partially failed. However it will always overestimate the volume unless the shape is convex. There are also a number of concave hulling

213 approaches. Some are mathematically defined such as AlphaShapes (Edelsbrunner \& Mücke, 214 1994) and Ball Pivoting (Bernardini et al., 1999) and require additional parameters defining the maximum level of permitted convexity. Others are proprietary and can require considerable manual intervention such as the built in hole-filling algorithms in Geomagic. This latter group provides the highest quality reconstructions but at the expense of considerable operator time. For this paper we concentrated on convex hulls under the assumption that the level of concavity in individual body segments was likely to be relatively small. The relative segment mass of all participants are reported in Fig. 3 (the segmented convex hulls are shown in Fig. S1 in the supporting information). Figure 3 also displays average values from literature. As the participants were imaged wearing shoes, the foot volume was overestimated significantly. It is possible to adjust the value using a foot-specific scaling factor that accounts for this overestimation although of course if the subsequent use of the BSP parameters is in experiments with participants wearing shoes then the shoe mass becomes an important part of the segment.

226 For the purpose of this paper, a scaling factor was derived based on a single participant (P5), by comparing the convex hull volume of the of the foot imaged in socks versus the convex hull volume wearing shoes, and this factor (of 0.51) applied to all participants' inertial values of the feet. The moments of inertia are shown in in Fig. 4 together with average values from literature. Geometric methods also allow us to calculate the products of inertia which are otherwise simply assumed to be zero. The average products of inertia are depicted in Fig. 5 (absolute values shown only, signed values reported in the supporting information Table S2-S4). Some segments, e.g. the 
233 thigh or trunk, have products of inertia that are of a similar order of magnitude as their moments 234 of inertia, which is indicative of a noticeable difference between the inertial principal axes and 235 the anatomical principal axes. The majority of the products of inertia are however significantly 236 smaller than the moments of inertia (of the same segment) by one to two orders of magnitude.

237 Figure 6 contains the relative centre of mass in the longitudinal segment direction, i.e. along the 238 z-axis with the exception of the foot whose longitudinal axis corresponds to the $\mathrm{x}$-axis (see Fig.

239 2). Figure 7 shows the shift of CoM from the longitudinal axis in the transverse plane ( $x-y$

240 plane). The CoM values in literature assume a zero shift from the principal anatomical

241 (longitudinal) axis. The shift values we found with our geometric method are generally unequal

242 to zero, but they have be to viewed with caution as the placement of the reference anatomical

243 axis itself has uncertainties associated with it. The numerical values presented in Fig. 3-7 and the

244 segment lengths are reported in the supporting information (Tables S2-S13)

246 To estimate the effect of the convex hull approximation on the mass estimation versus the

247 original body segment shape, the volumes of a high resolution 3D body scan and of their convex

248 hull approximation were calculated and compared. A detailed surface mesh was obtained from

249 the National Library of Medicine's Visible Human Project (Spitzer et al., 1996) by isosurfacing

250 the optical slices using the VTK toolkit (http://www.vtk.org) and cleaning up the resultant mesh

251 using Geomagic. The surface mesh of the 3D body scan was separated into body segments and

252 the volume calculated following the same methodology as used for the point cloud data. A

253 convex hull was applied to each body segment and the volume calculated again (see Fig. 8). The

254 volume overestimations for each body segment (averaged between left and right) are shown Fig.

2559 (column $\mathrm{CH}$ ). Several body segments showed a large relative volume overestimation (using

$25610 \%$ error as a cutoff, although the choice would depend on the required accuracy): foot (26\%),

257 shank (31\%), hand (47\%) and forearm (16\%). This is due to the relatively strong curvatures in

258 these segments. To minimize the effect, these body segments were subdivided (see Fig. 10) and

259 the convex hulls recalculated. The results of the divided segments are also shown in Fig. 9

260 (column CHD), and the decrease in volume overestimation is apparent. The volume

261 overestimation of the subdivided foot (11\%), shank (11\%) and forearm (5\%) are at a similar

262 level to the other body segments and would probably be acceptable in many cases. The hands

263 show the largest relative mass overestimation still (25\%), which is due to its curved position and

264 slightly open fingers. The convex hull error of the hand is, however, expected to be significantly 
265 smaller if the hand is imaged while being held in a straight position with no gaps between the 266 digits.

268 Figure 11 contains the relative mass estimations of the original surface mesh, the convex hulls 269 with and without subdivision, and the average and regression model values found in literature.

270 With a BMI value of almost 28, the male visible human is not well represented by the average or 271 regression model values found in literature, where the majority of the studies involve relatively 272 athletic people (BMI average of around 24) or obese individuals (BMI over 30). The convex

273 hulls of the subdivided segments (CHD in Fig. 11) give the closest approximation to the original 274 mesh and, with the exception of the hands, are within a relative error of less than 5\%. The 275 relative error of the convex hull of the whole segments ( $\mathrm{CH}$ in Fig. 11) is larger but closer to the 276 original mesh than average and regression values given in literature. The moments of inertia are 277 overestimated as well as they are a product of the mass of the segment. Their overestimation 278 follows the same trend as the mass overestimation, i.e. the largest overestimation occurs for the 279 hands, followed by the shanks and feet (see Fig. S2 in supporting information), and the 280 subdivided segments produce more accurate values with an average relative error below $10 \%$.

\section{Discussion}

We can see from the results that the proposed methodology produces values that are very similar to those derived using regression equations. There are no consistent problems although it is clearly important that the hand is held in a suitable flat position but with fingers adducted so that the hulling can provide an accurate volume estimation. We would expect that the photogrammetric process will work as well as any of the published geometrical approaches (Hanavan, 1964; Hatze, 1980) since it is simply an automated process for achieving the same outcome. The procedure is currently moderately time consuming in total but the interaction time with the participant is extremely short and involves no contact which can be very beneficial for certain experimental protocols or with specific vulnerable participants. Since most of the time is spent post-processing the data, we expect that this post-processing could be streamlined considerably by writing dedicated software rather than the current requirement of passing the

296 data through multiple software packages.

297 In general regression equations work well for applicable populations and are probably more 
298 suitable if body mass distribution is not a major focal point of the research, particularly given

299 that in some cases it can be shown that experimental outcomes are not especially sensitive to the

300 BSP parameters chosen (Yokoi et al., 1998). The values generated in our sample are relatively

301 close to those generated by using regression equations but BSP values are highly variable

302 between individuals and current regression equations are only suitable for a very limited range of

303 body shapes. This is particularly the case when we are dealing with non-standard groupings such

304 as children, the elderly or people with particularly high or low BMI values.

However there are some specific issues with this technique that could to be improved for a more streamlined and potentially more accurate workflow (see Fig.12, which summarises the steps involved in estimating body segment parameters using photogrammetry). Convex hulling of the point cloud is a robust and fast way to produce surface meshes. The fact that it systematically overestimates the volume of concave features can be improved by subdividing body segments into smaller parts and the decision then becomes what level of subdivision is appropriate for an acceptable level of accuracy (see Fig. 12 C). For example, with only one subdivision of the shank and forearm the relative error of their volume overestimation was reduced by a factor of three, and the end result was within $10 \%$ of the true value which is probably sufficient in most cases, especially given the level of uncertainty in other parameters such as segment specific density. It is important to note that the scaling factor used in our method minimises the segment mass estimation errors introduced by the volume overestimation significantly. In fact, if all hulled segment masses (i.e. the product of segment volume and segment-specific density, see Fig. 12 D) were overestimated by $10 \%$, the final body segment mass would be calculated correctly due to the scaling factor applied to each segment. Using a pro-rata scaling factor therefore performs best when the relative errors of the volume estimation of each segment are within a small range of each other.

The adoption of one of the concave hulling techniques is likely to lead to a similar level of improvement again with a minimum (but not zero) level of additional work. The level of subdivision required not only depends on the body segment, but also the population studied so it may well be appropriate that the segmentation level is adjusted according to the type of study and its sensitivity to inaccuracies in the BSP (i.e. multiple segment subdivisions increase accuracy of volume estimation). In this work, a uniform scaling factor and constant body density (apart from the trunk) was assumed. It is well known that the density varies among body 
331 segments as well as among populations due to different percentages of fat and muscle tissue

332 (Drillis, Contini \& Bluestein, 1964; Durnin \& Womersley, 1974; Zatsiorsky, 2002). Thus, using

333 segment and population specific densities (and scaling factors) may improve the accuracy of the

334 presented methodology if such values are available or derived. Similarly important contributions

335 to segmental mass distribution such as the presence of the lungs within the torso can be modelled

336 explicitly which may lead to small but important shifts in the centre of mass (Bates et al., 2010).

338 In terms of technology, the current arrangement of using 18 Raspberry Pi cameras is reasonably

339 straightforward and relatively inexpensive. It requires no calibration before use, and the process

340 of moving the subject into the target area is extremely quick. However it does take up a great

341 deal of room in the laboratory and the current software is reliant on clothing contrast for the

342 reconstructions which limits the flexibility of the technique. One area where this could be

343 improved is by projecting a structured light pattern onto the subject so that areas with minimal

344 contrast can be reconstructed accurately (Casey, Hassebrook \& Lau, 2008). Our results show that

34518 cameras is currently the minimum needed for full body reconstruction and a system with 36

346 or more cameras would produce better point cloud reconstruction results by minimizing areas of

347 potential occlusions (such as between the legs or between the arms and trunk) and increasing the

348 point cloud density. To what degree a more densely packed point cloud would significantly

349 improve the accuracy of the estimated inertial parameters based on convex hulls would be an

350 interesting aspect to investigate further. We would expect a denser point cloud to facilitate the

351 use of more complex meshing methods instead of convex hulling.

353 One future use of this technology is clearly the use of such systems and algorithms for complete

354 motion capture (Sellers \& Hirasaki, 2014). The limitation currently is that these cameras would

355 need to be closely synchronised and whilst the proposed system is adequate for producing a

356 single still image, it is currently not able to adequately synchronise video. In addition the video

357 resolution is much lower and this makes the reconstruction more difficult. However we predict

358 that markerless, multiple video camera structure from motion systems will become a much more

359 common mainstream tool for experimental motion capture in the near future. Ideally we could

360 imagine that such a system would both do the motion capture and also the body segment

361 parameter reconstruction since much of the computational technology would be shared.

\section{Conclusion}


365 A methodology based on structure form motion photogrammetric reconstruction has been

366 presented that provides subject-specific body segment parameters. The method relies on the

367 surface depth information extracted from multiple photographs of a participant, taken

368 simultaneously from multiple different view points. The brief interaction time with the

369 participants (taking all required photos simultaneously, and measuring the height and weight

370 only) makes this a promising method in studies with vulnerable subjects or where cost or ethical

371 constraints do not allow the use of other imaging methods such as CT or MRI scans. Unlike

372 regression models that are valid only for a small population sample, we expect the proposed

373 methodology to be able to perform equally well for a wide range of population samples. The

374 post-processing time is lengthy compared to using regression models or average values from

375 literature but not compared to processing MRI or CT data. The 3D scanner presented in this

376 paper was able to produce a sufficient 3D data points to estimate body segment volumes with

377 only 18 RPi cameras, which kept the hardware cost to a minimum. Depending on the accuracy

378 required for the project, we would expect both more cameras and higher resolution cameras to

379 improve the robustness of the 3D point cloud reconstruction.

380 While the results presented in this work were derived using commercial software, such as

381 AgiSoft, Geomagic and Matlab ${ }^{\circledR}$, we were able to to achieve similar results using open-source

382 software only (such as VisualFMS (http://ccwu.me/vsfm/) for calculating 3D point clouds and

383 MeshLab (http://meshlab.sourceforge.net/) for point cloud segmentation, hulling and BSP

384 calculation). This makes our proposed methodology, in combination with the low hardware

385 costs, particularly promising for small-budget projects.

386

387

\section{Acknowledgements}

The authors would like to thank Dave Jones for the development of the Compound Pi programme and his generous help with the network setup of the Raspberry Pi scanner.

\section{References}

Agarwal S, Snavely N, Simon I, Seitz SM, Szeliski R. 2009. Building Rome in a day. IEEE Int. Conf. on Computer Vision:72-79. 
Bates KT, Manning PL, Margetts L, Sellers WI. 2010. Sensitivity analysis in evolutionary robotic simulations of bipedal dinosaur running. Journal of Vertebrate Paleontology 30:458-466.

Bauer JJ, Pavol MJ, Snow CM, Hayes WC. 2007. MRI-derived body segment parameters of children differ from age-based estimates derived using photogrammetry. Journal of biomechanics 40:2904-10.

Bernardini F, Mittleman J, Rushmeier H, Silva C, Taubin G. 1999. The Ball-Pivoting Algorithm for Surface Reconstruction. IEEE Transactions on Visualization and Computer Graphics 5:349-359.

Bonnechère B, Jansen B, Salvia P, Bouzahouene H, Sholukha V, Cornelis J, Rooze M, Van Sint Jan S. 2014. Determination of the precision and accuracy of morphological measurements using the KinectTM sensor: comparison with standard stereophotogrammetry. Ergonomics 57:622-31.

Brožek J, Grande F, Anderson JT, Keys A. 1963. Densitometric Analysis of Body Composition: Revision of some Quantitative Assumptions. Annals of the New York Academy of Sciences 110:113-140.

Casey CJ, Hassebrook LG, Lau DL. 2008. Structured light illumination methods for continuous motion hand and face-computer interaction. In: Asai K ed. Human Computer Interaction: New Developments. InTech, 297-308.

Clarkson S, Choppin S, Hart J, Heller B, Wheat J. 2012. Calculating Body Segment Inertia Parameters from a Single Rapid Scan Using the Microsoft Kinect. Proceedings of the 3rd International Conference on 3D Body Scanning Technologies, Lugano, Switzerland, 16-17 October 2012:153-163.

Clauser CEC, McConville JJT, Young JWJ. 1969. Weight, volume, and center of mass of segments of the human body. Antioch Coll Yellow Springs OH.

Dempster W. 1955. Space requirements of the seated operator: geometrical, kinematic, and mechanical aspects of the body, with special reference to the limbs. Wright-Patterson Air Force Base Ohio.

Drillis R, Contini R, Bluestein M. 1964. Body segment parameters. Artificial limbs 8:44-66. Durnin J, Womersley J. 1974. Body fat assessed from total body density and its estimation from skinfold thickness: measurements on 481 men and woman aged from 16 to 72 years. British Journal of Nutrition 32:77-97.

Edelsbrunner H, Mücke EP. 1994. Three-dimensional alpha shapes. ACM Transactions on Graphics 13:43-72.

Falkingham PL. 2012. Acquisition of high resolution three-dimensional models using free, opensource, photogrammetric software. Palaeontologia Electronica 15:1T:15p. 
431 Furukawa Y, Ponce J. 2010. Accurate, dense, and robust multiview stereopsis. IEEE transactions on pattern analysis and machine intelligence $32: 1362-76$.

433 Garsthagen R. www.pi3dscan.com.

434 Hanavan EP. 1964. A Mathematical Model of the human body. Air force aerospace medical 435 research lab Wright-Patterson AFB Ohio.

436 Hatze H. 1980. A mathematical model for the computational determination of parameter values of 437 anthropomorphic segments. Journal of Biomechanics 13:833-843.

Hobson J. http://hackaday.com/2014/03/07/an-affordable-full-body-studio-grade-3d-scanner/. Jensen R. 1978. Estimation of the biomechanical properties of three body types using a photogrammetric method. Journal of Biomechanics 11:349-358.

Leva P De. 1996. Adjustment to Zatsiorsky-Seluyanov's segment inertia parameters. J. Biomech. 29:1223-30.

Lowe DG. 1999. Object recognition from local scale-invariant features. Proceedings of the Seventh IEEE International Conference on Computer Vision 2:1150-1157.

Martin P, Mungiole M, Marzke M, Longhill J. 1989. The use of magnetic resonance imaging for measuring segment inertial properties. Journal of Biomechanics 22:367-376.

McCarthy J. 2014. Multi-image photogrammetry as a practical tool for cultural heritage survey and community engagement. Journal of Archaeological Science 43:175-185.

McConville J, Clauser C, Churchill T. 1980. Anthropometric relationships of body and body segment moments of inertia. Anthropology research project inc Yellow Springs Ohio.

Pearsall DJ, Reid JG, Livingston L a. 1996. Segmental inertial parameters of the human trunk as Mungiole M, Martin PE. 1990. Estimating segment inertial properties: comparison of

Pearsall D, Reid J, Ross R. 1994. Inertial properties of the human trunk of males determined from Multi-View Stereo Reconstruction Algorithms. 2006 IEEE Computer Society Conference on Computer Vision and Pattern Recognition - Volume 1 (CVPR'06) 1:519-528.

Sellers WI, Hirasaki E. 2014. Markerless 3D motion capture for animal locomotion studies. Biology open 3:656-668.

463

Sheets AL, Corazza S, Andriacchi TP. 2010. An automated image-based method of 3D subject- 
465

466

467

468

469

470

471

472

473

474

475

476

477

478

479

480

481

482

Siri WE. 1961. Body composition from fluid spaces and density: analysis of methods. In: Brožek J, Henschel A eds. Techniques for measuring body composition. Washington DC: National Academy of Sciences - National Research Council, 223-44.

Spitzer V, Ackerman MJ, Scherzinger AL, Whitlock D. 1996. The visible human male: a technical report. Journal of the American Medical Informatics Association 3:118-30. Straub J, Kerlin S. 2014. Development of a Large, Low-Cost, Instant 3D Scanner. Technologies 2:76-95.

Triggs B, McLauchlan P, Hartley RI, Fitzgibbon AW. 2000. Bundle adjustment — a modern synthesis. Lecture Notes in Computer Science 1883:298-372.

Weinbach A. 1938. Contour maps, center of gravity, moment of inertia and surface area of the human body. Human Biology 10:356-371.

Winter DA. 1979. Biomechanics of Human Movement. New York: Wiley and Sons.

Yeadon M. 1990. The simulation of aerial movement-II. A mathematical inertia model of the human body. Journal of Biomechanics 23:67-74.

Yokoi T, Takahashi A, Okada H, Ohyama KB, Muraoka M. 1998. Is the Selection of Body Segment Interia Parameters Critical to the Results of the Kinematic and Kinetic Analysis of Human Movement? Anthropological Science 106:371-383.

Zatsiorsky VM. 2002. Kinetics of HumanMotion. Human Kinetics. 
1

Body Scanner Design.

A: Point cloud reconstruction with varying number of cameras. B: Schematic representation of the RPi scanner design.

A

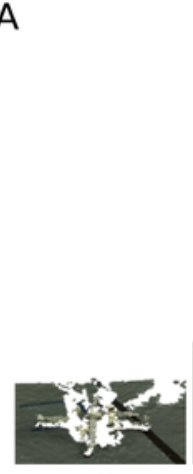

9

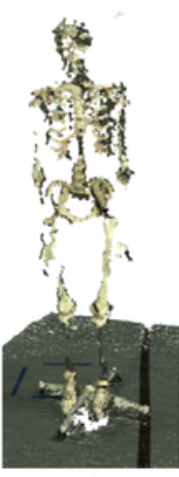

12

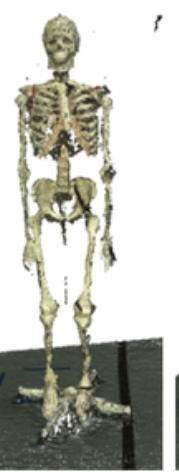

18

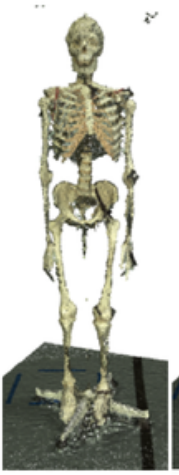

24

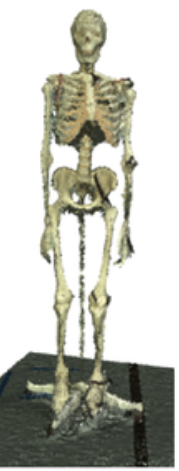

36

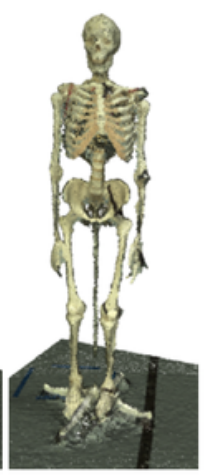

72

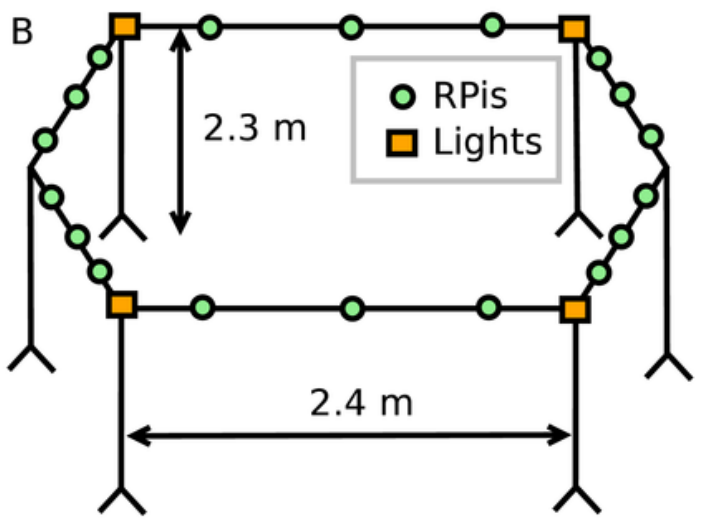




\section{2}

Image processing work flow.

Images from the RPI scanner are converted to 3D point clouds which are then scaled and segmented manually. Subsequently, convex hulling is used to produce a surface mesh around each body segment.

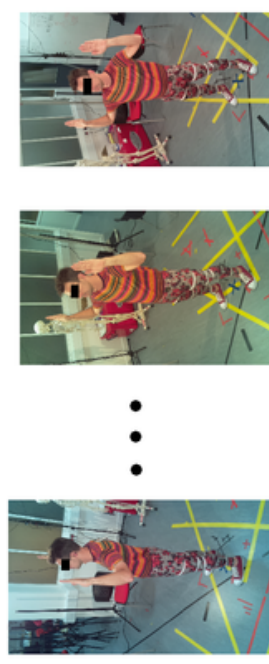

Raw images
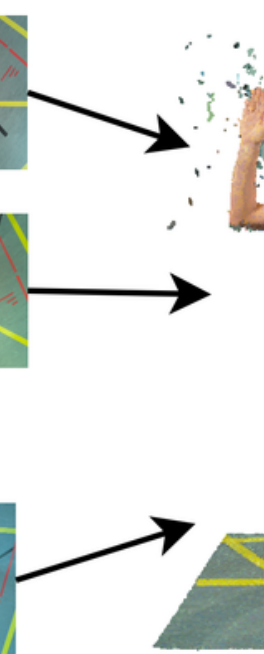

3D point cloud

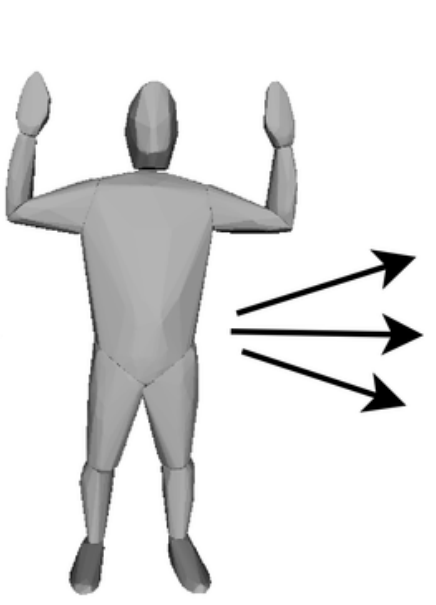

Segmented convex hull

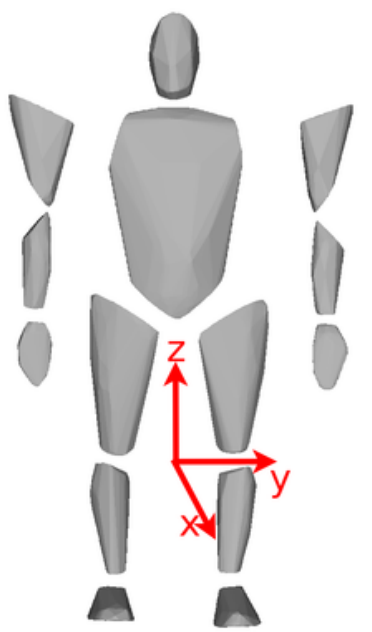

Segments in standard pose 


\section{3}

Segment mass (as $\%$ of body mass).

P: Average value of all six participants (error bars show standard deviation). Foot mass adjusted by a factor of 0.51 to compensate for volume overestimation due to wearing shoes. $Z(m)$ : Male average values reported by Zatsiorsky. $Z(f)$ : Female average values reported by Zatsiorsky (Leva, 1996; Zatsiorsky, 2002). D(m): Male average values by Dempster (via Zatsiorsky) (Dempster, 1955; Zatsiorsky, 2002).

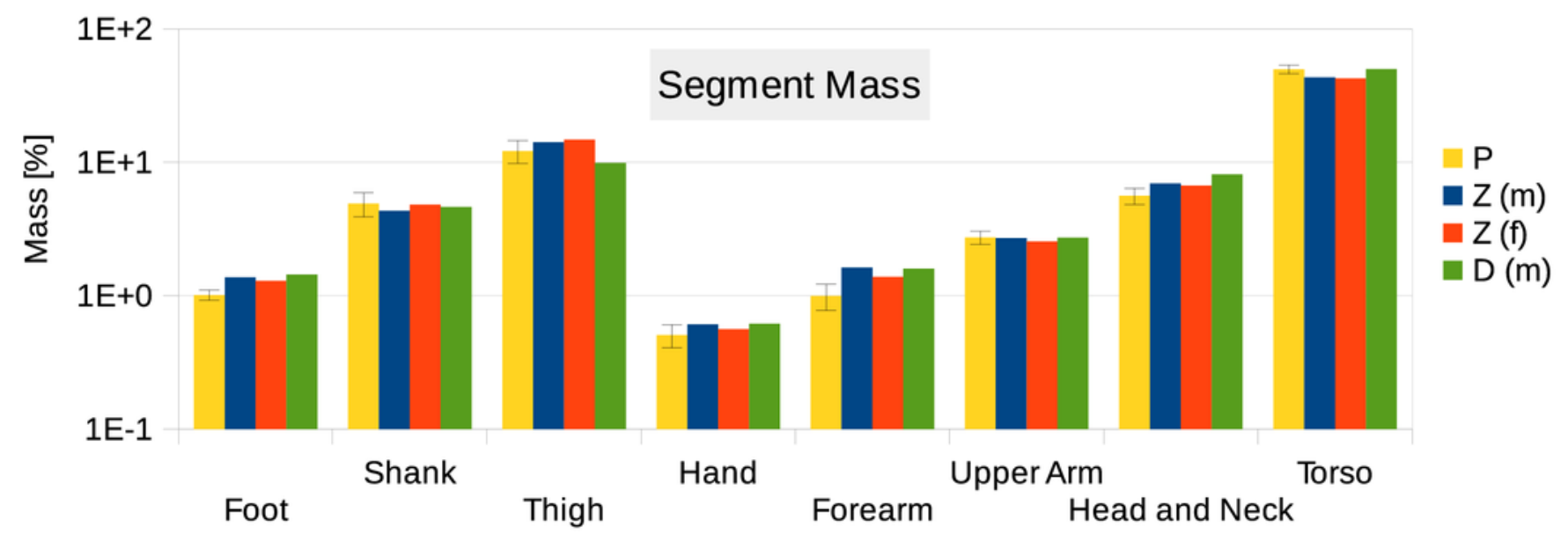




\section{4}

Moment of inertia in $\left[10^{4} \mathrm{~kg}^{*} \mathrm{~m}^{2}\right]$.

P: Average value of all six participants (error bars show standard deviation). Foot moment of inertia adjusted by a factor of 0.51 to compensate for volume overestimation due to wearing shoes. $Z(m)$ : Male average values reported by Zatsiorsky. $Z(f)$ : Female average values reported by Zatsiorsky (Leva, 1996; Zatsiorsky, 2002). The definition of the coordinate system is shown in Fig. 2.

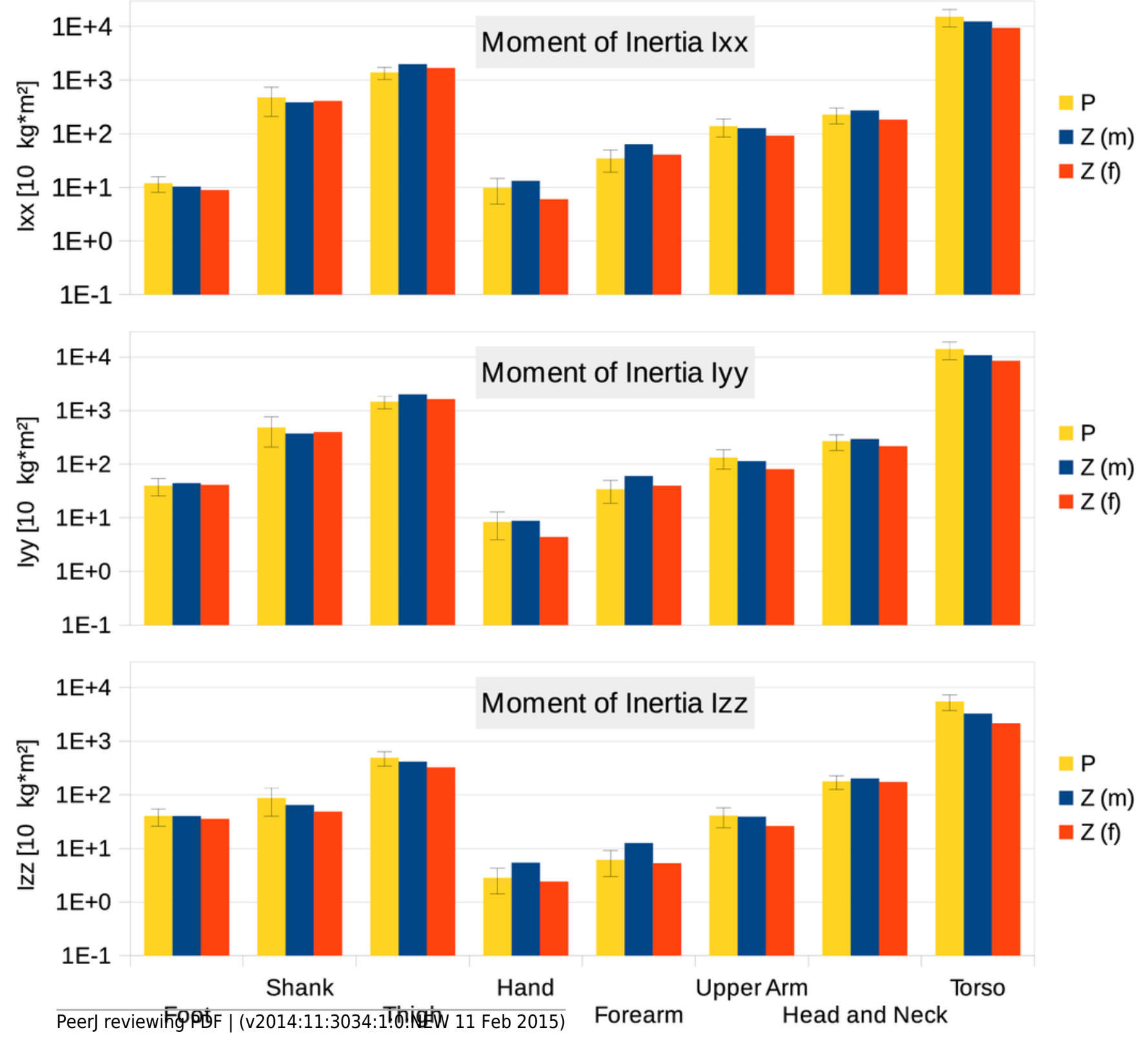




\section{5}

Absolute values of products of inertia in $\left[10^{4} \mathrm{~kg}^{*} \mathrm{~m}^{2}\right]$.

he absolute values of Ixy, Ixz and lyz are shown together with a positive error bar (negative error bar is symmetrical) equal to one standard deviation. The signed values are reported in the supporting information in Tables S2-S4. The Ixy value of the hand is smaller than $10^{3}$ $\mathrm{kg}^{*} \mathrm{~m}^{2}$ and is not displayed. Foot products of inertia adjusted by a factor of 0.51 to compensate for volume overestimation due to wearing shoes.

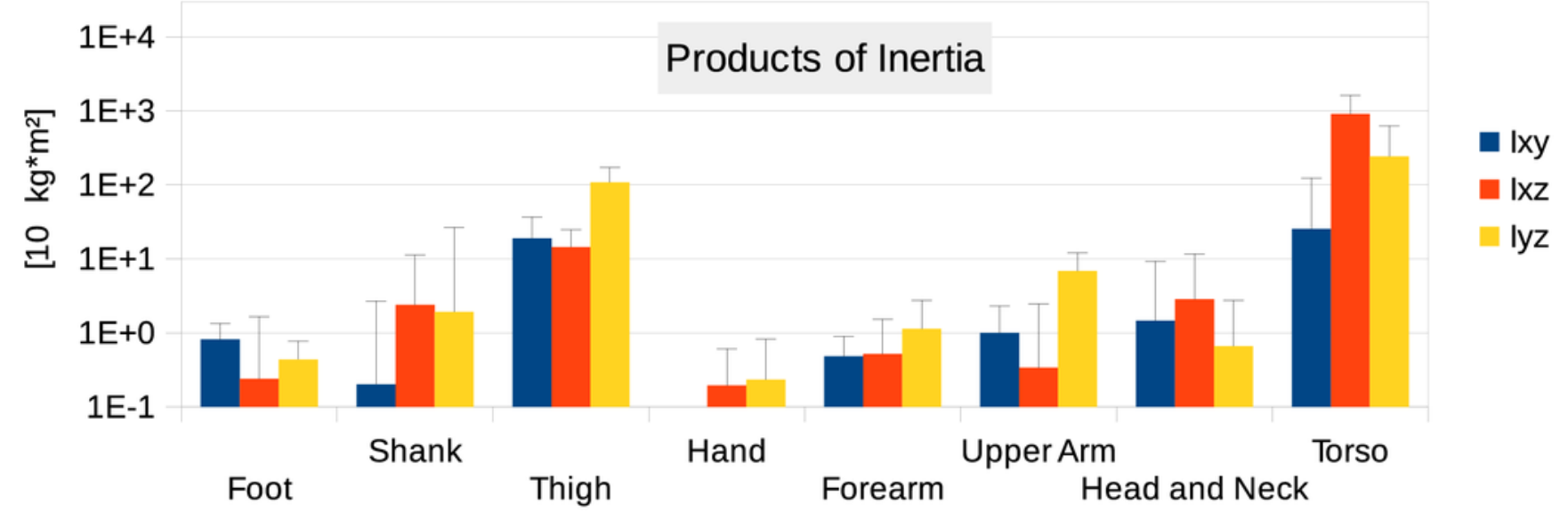




\section{6}

Centre of mass along the longitudinal axis.

P: Average value of all six participants (error bars show standard deviation). Z(m: male, $f$ : female): Average values by Zatsiorsky, adjusted by de Leva. The CoM is given as $\%$ of the segment length. The definition of the segments and reference points are given in the supporting information Table S1 - Exceptions: * Foot of participants: Heel and toe end point of participant's shoes instead of foot. ** Forearm and Upper Arm of Z: Elbow reference point is the elbow joint centre instead of the Olecranon (Leva, 1996; Zatsiorsky, 2002).

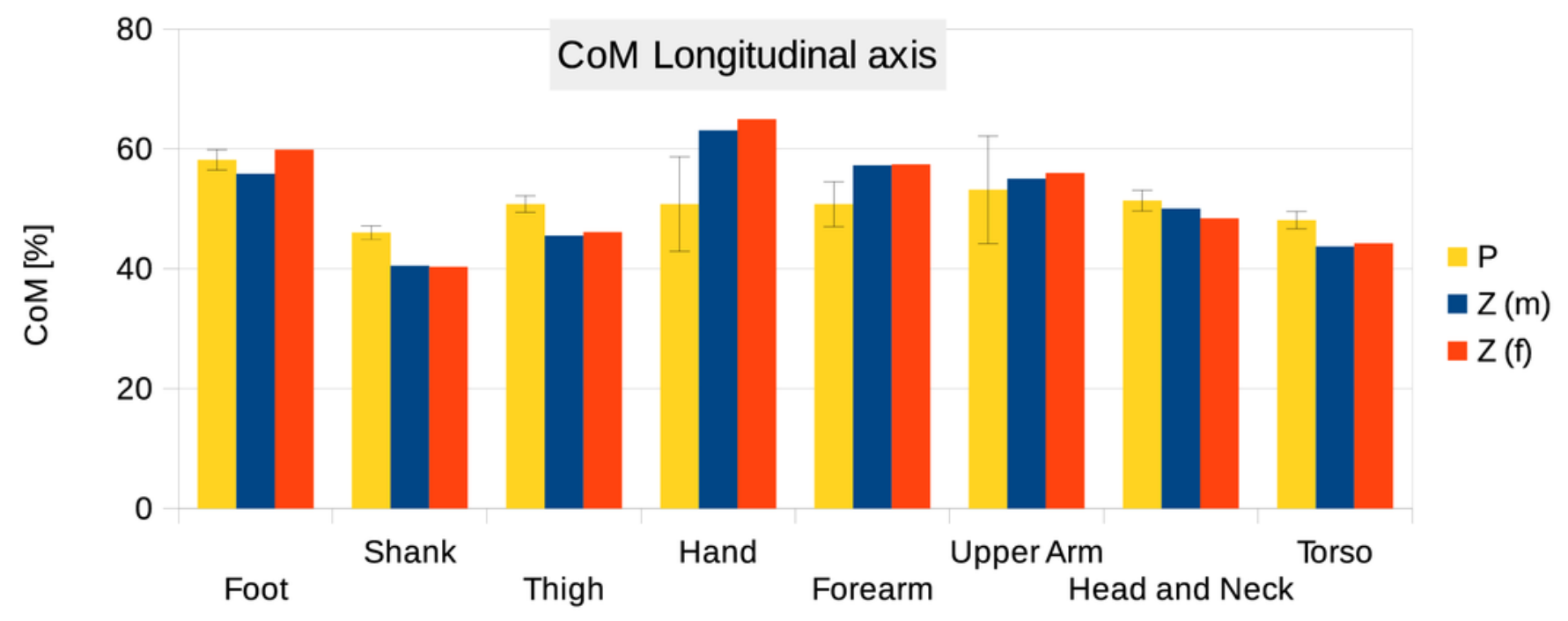




\section{7}

CoM shift from the anatomical longitudinal axis in the transverse $(x-y)$ plane.

Average values of all six participants are shown (error bars show standard deviation). Due to mirror-symmetry, the y-values of the segments on the left- and right-hand side have opposite signs. To calculate the average, the sign of the segments on the left-hand side was inverted. The CoM is given as \% of the segment length. The data of the foot is not included due to the participants wearing shoes.

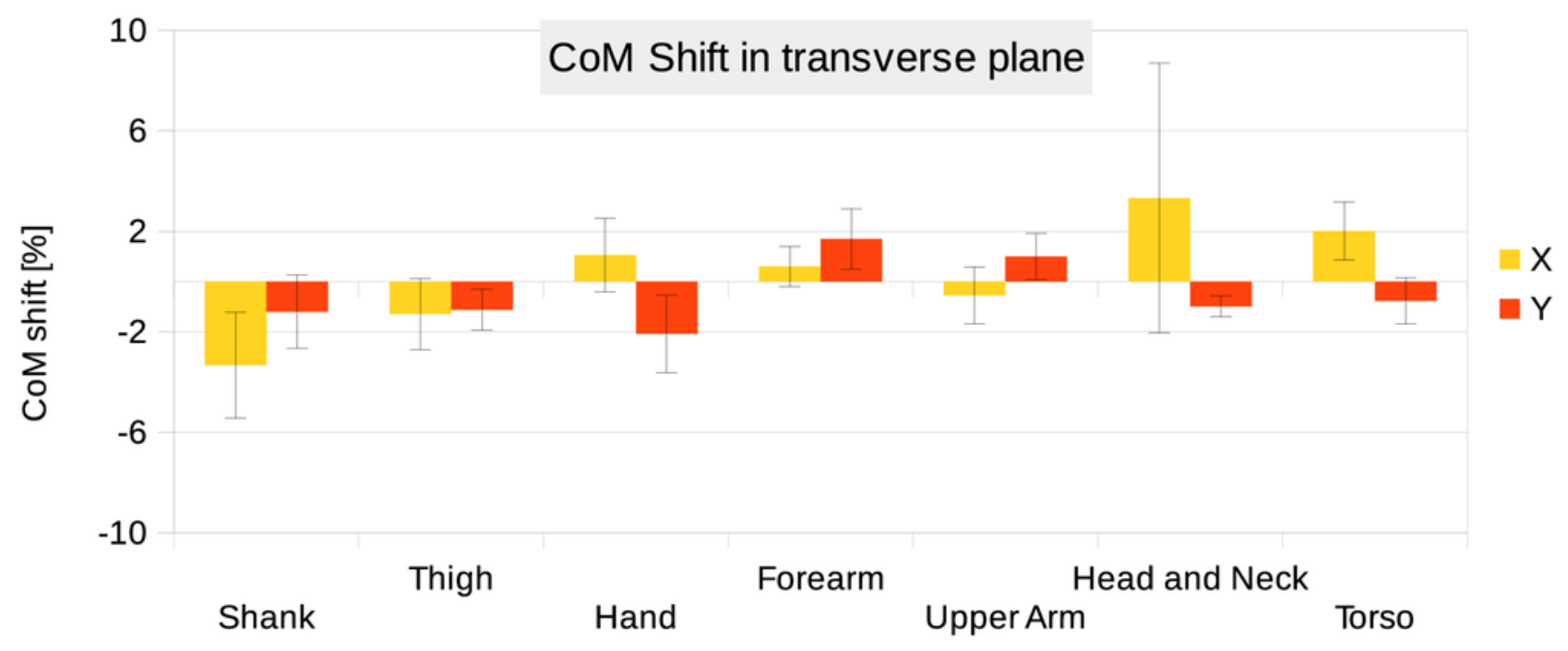


8

Visible Human surface mesh.

A: High-resolution surface mesh. B: Convex hull mesh.

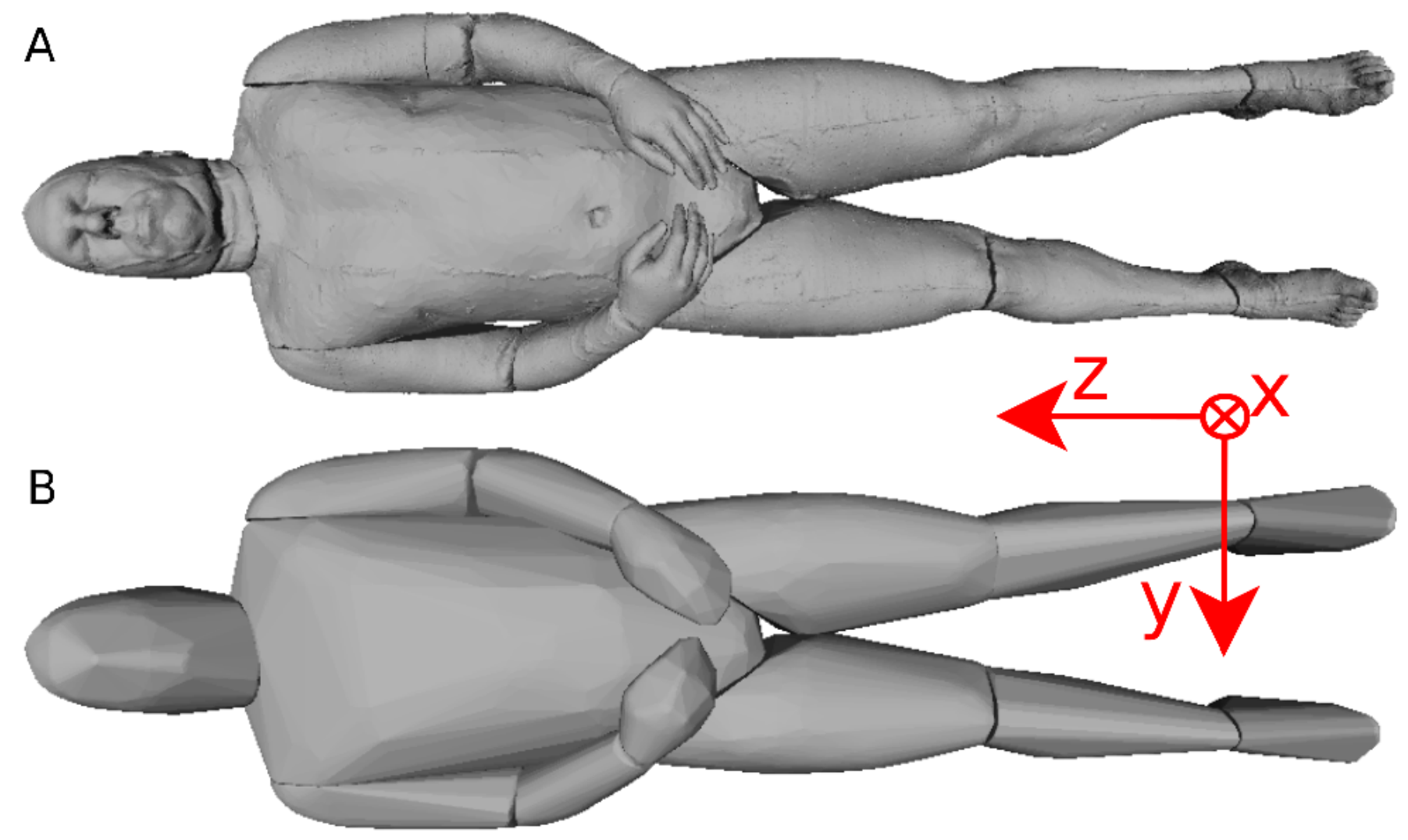


9

Segment volume overestimation of the hulled mesh versus the high-resolution surface mesh of the Visible Human.

Data shown as the relative difference of the hull with respect to the original mesh. $\mathrm{CH}$ :

Convex hull of body segment. CHD: Convex hull of divided body segments (only segments indicated with an * were subdivided, see Fig. 10).

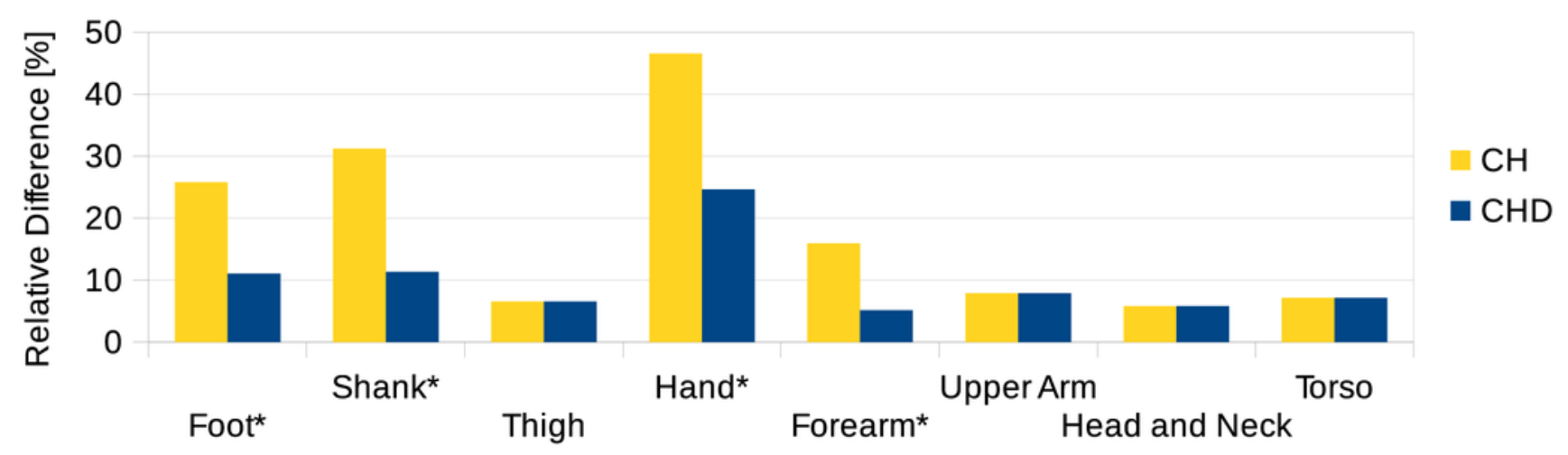




\section{0}

Subdivision of the body segments with large curvature.

The first row (S) shows the high-resolution surface mesh, the second row $(\mathrm{CH})$ the convex hull of the whole body segment, and the bottom row (CHD) the convex hulls of the subdivided body segments.

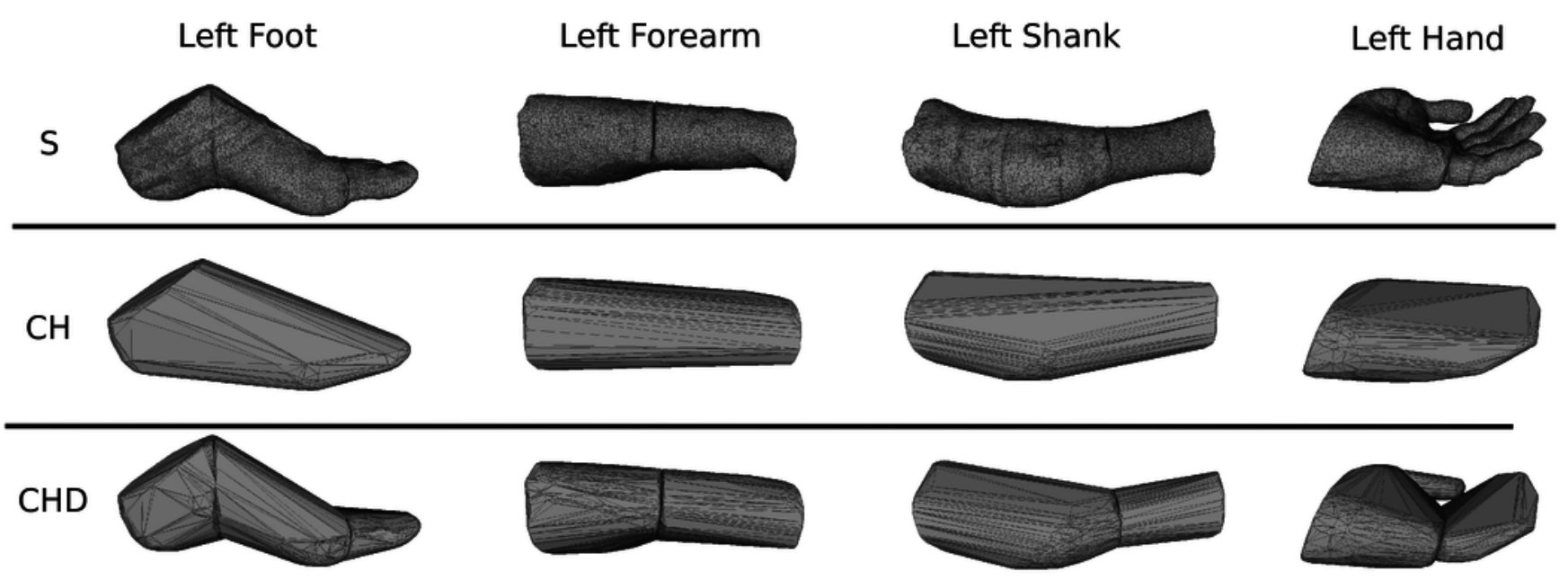




\section{1}

Male Visible Human segment mass (as \% of body mass) of the high-resolution mesh, convex hull, regression model and average values.

S: High-resolution surface mesh. $\mathrm{CH}$ : Convex Hull of whole body segments. CHD: Convex Hull with subdivided body segments (only segments indicated with an * were subdivided as shown in Fig. 10). ZR: Values predicted using Zatsiosrky's linear regression model (using weight and height). Z: Male average values reported by Zatsiorsky. D: Male average values reported by Dempster (Dempster, 1955; Leva, 1996; Zatsiorsky, 2002).

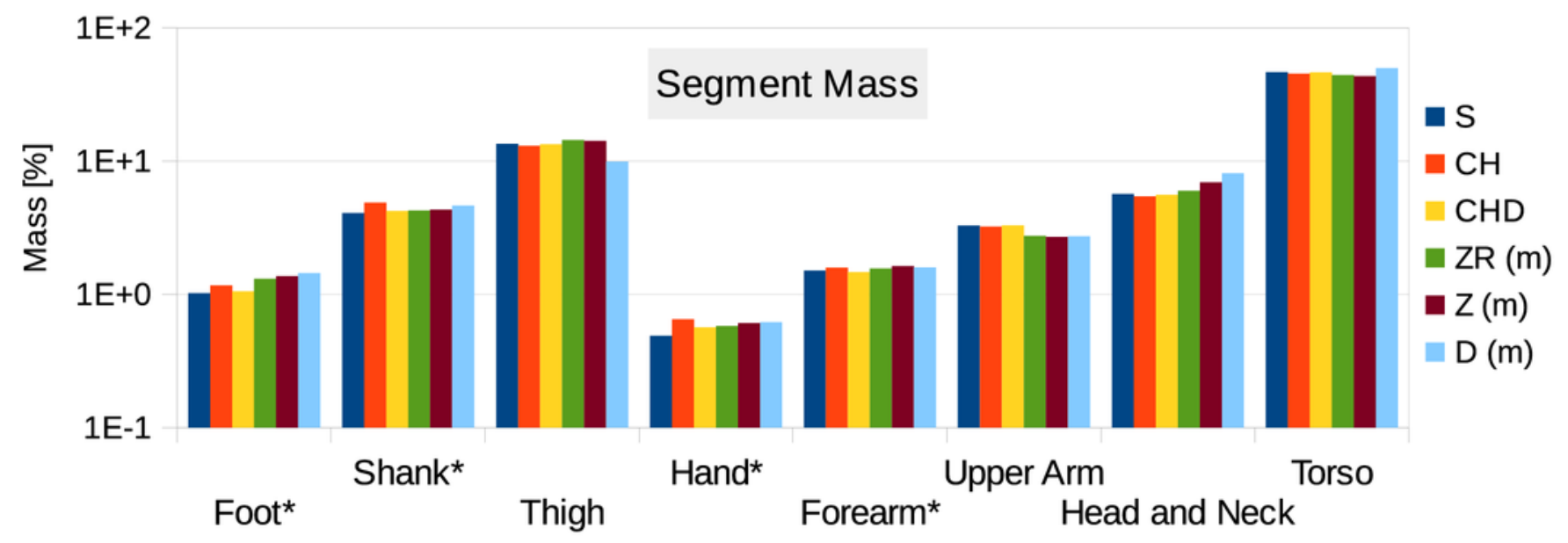


12

Methodology to estimate subject-specific body segment parameters using photogrammetry.

A. Photogrammetry; B. Body segmentation; C. Segment hulling; D. Inertial parameter estimation. 


\section{A. Photogrammetry:}

Surface point cloud reconstruction
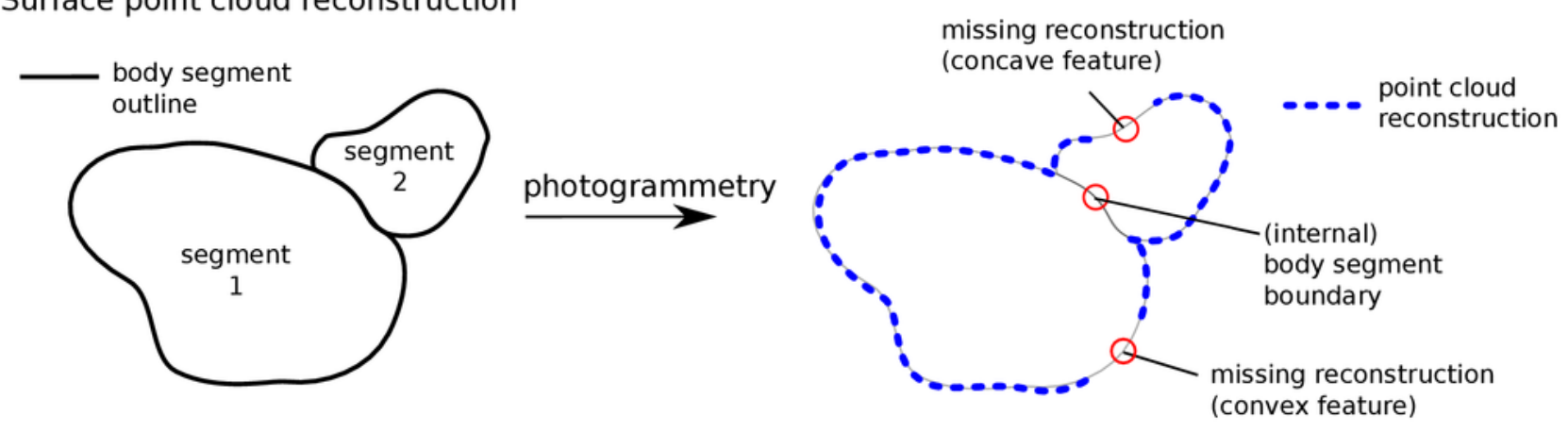

\section{B. Body segmentation:}

Separation of point cloud into body segments
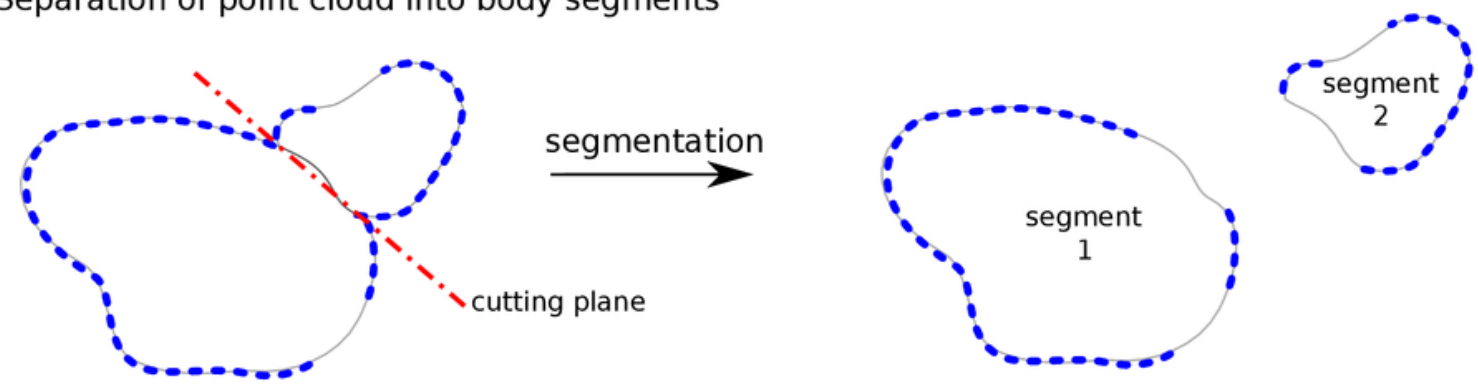

\section{Segment hulling:}

Creation of closed surface mesh using hulling algorithms

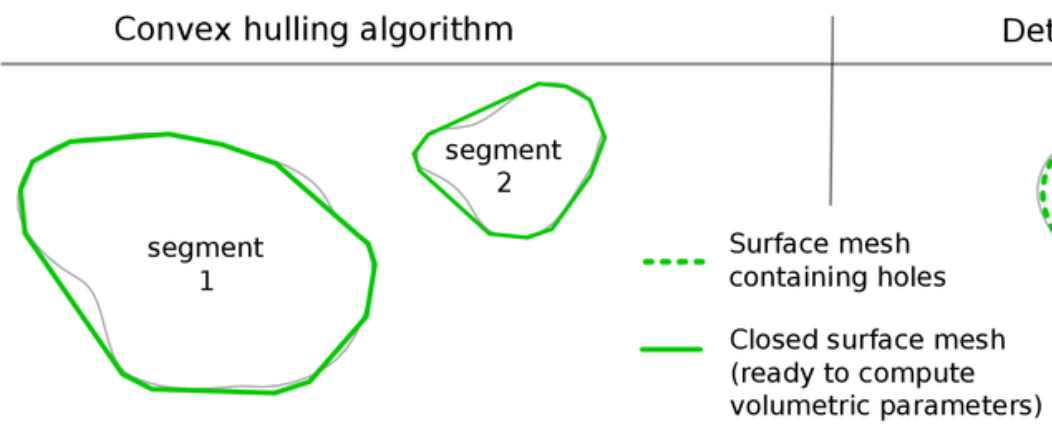

Detailed surface meshing algorithm
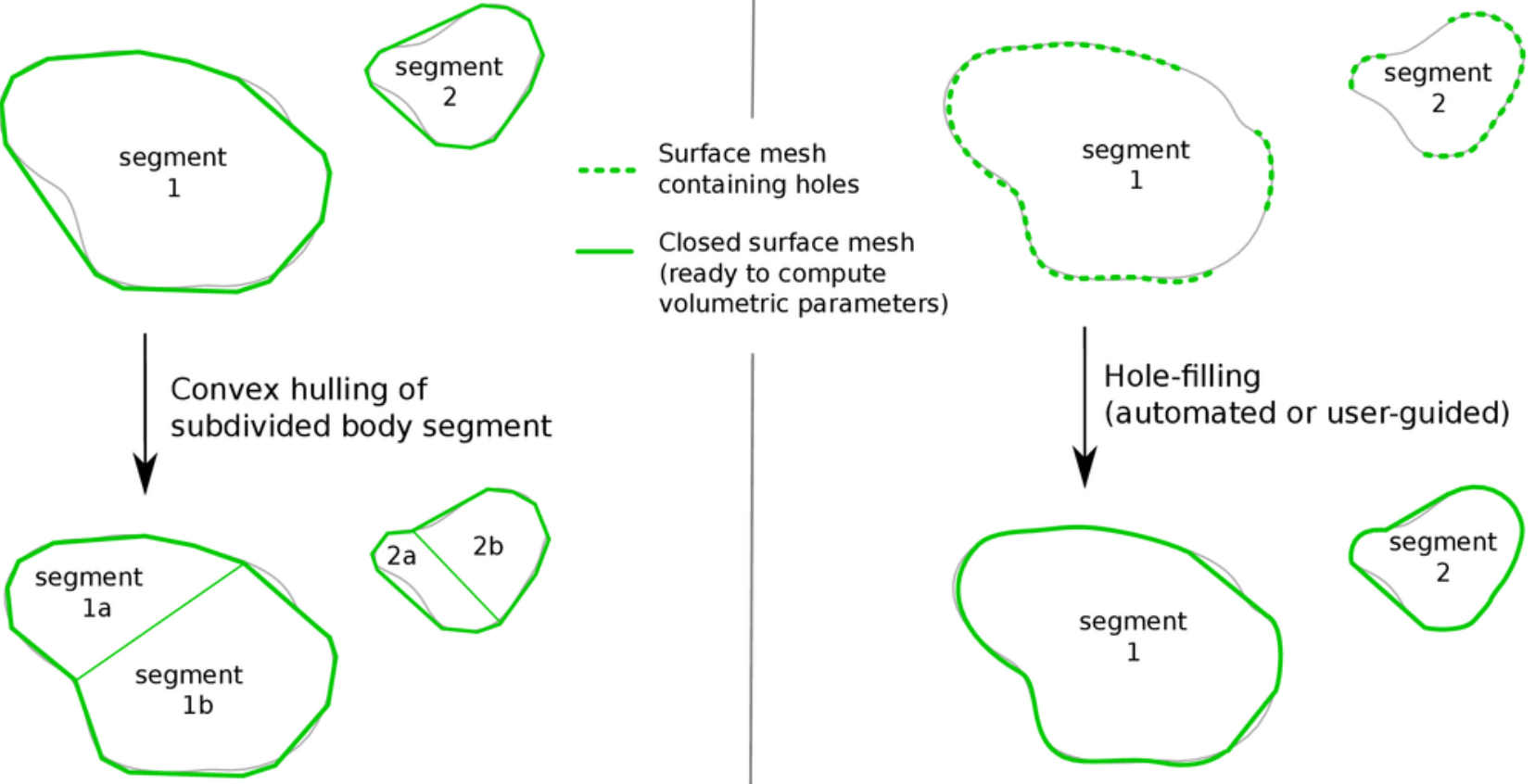

\section{Inertial parameter estimation}

Estimation of subject inertial segment parameters using body segment densities and scaling factor

(1) Hull mass $=$ Hull volume $\times$ Segment density

(2) Scaling factor $=\frac{\text { Subject mass }}{\text { Sum of all hull masses }}$ Peer) reviewing PDF | (v2014:11:3034:1:0:NEW 11 Feb 2015)
(3) Segment mass $=$ Hull mass $\times$ Scaling factor

(4) Segment inertial tensor $=$ Hull inertial tensor $\times$ Segment density $\times$ Scaling factor 


\section{Table $\mathbf{1}$ (on next page)}

Participant mass and weight.

P1 - P6: Participants (m: male, f: female). VH: Male Visible Human. 


\section{PeerJ Reviewing Manuscript}

\begin{tabular}{l|l|l|l|l|l|l|l} 
& P1 (m) & P2 (m) & P3 (m) & P4 (m) & P5 (f) & P6 (f) & VH (m) \\
\hline Mass $[\mathrm{kg}]$ & 73.4 & 77.0 & 88.2 & 87.8 & 65.4 & 55.2 & 90.3 \\
\hline Height $[\mathrm{m}]$ & 1.81 & 1.83 & 1.85 & 1.83 & 1.65 & 1.58 & 1.80
\end{tabular}

